Discrete Comput Geom 37:139-162 (2007)

DOI: $10.1007 / \mathrm{s} 00454-006-1285-4$

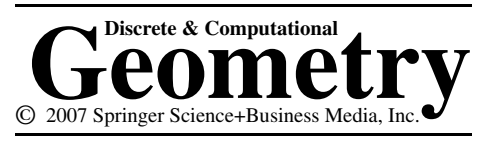

\title{
Realizability of Graphs in Three Dimensions*
}

\author{
Maria Belk \\ Department of Mathematics, Texas A\&M University, \\ College Station, TX 77843, USA \\ mbelk@math.tamu.edu
}

\begin{abstract}
This paper concludes the characterization of 3-realizable graphs begun by Belk and Connelly [BC1]. A graph is 3-realizable if, for every configuration of its vertices in $\mathbb{E}^{N}$ with $N \geq 3$, there exists a corresponding configuration in $\mathbb{E}^{3}$ with the same edge lengths. In this paper the two graphs $V_{8}$ and $C_{5} \times C_{2}$ are shown to be 3-realizable. As shown by Belk and Connelly [BC1], this means that the forbidden minors for 3-realizability are $K_{5}$ and $K_{2,2,2}$.
\end{abstract}

\section{Introduction}

A graph $G$ is a finite set of vertices $V(G)=\{1, \ldots, n\}$ and a finite set of edges $E(G)$, where each edge is a set containing exactly two vertices. The graphs we consider do not contain loops or multiple edges. The complete graph on $n$ vertices, denoted by $K_{n}$, is the graph with $n$ pairwise adjacent vertices. A good reference on graph theory is [D].

A realization of a graph $G$ is a function which assigns to each vertex $i$ of $G$ a point $p_{i}$ in some Euclidean space. When we draw a realization, we generally also draw the edges between vertices as straight lines. Note that a realization is different from an embedding, since the word embedding is usually reserved for the case when there are no self-intersections. For example, two vertices may be assigned to the same point in a realization, and edges may intersect and even overlap.

We say a graph $G$ is $d$-realizable if, given any realization $p_{1}, \ldots, p_{n}$ of the graph in some finite-dimensional Euclidean space, there exists a realization $q_{1}, \ldots, q_{n}$ in $\mathbb{E}^{d}$ with the same edge lengths: $\left|p_{i}-p_{j}\right|=\left|q_{i}-q_{j}\right|$ for all $\{i, j\} \in E(G)$. Note that this definition of $d$-realizability is a property of graphs-for a graph to be $d$-realizable, every realization of the graph must have a realization in $\mathbb{E}^{d}$.

* This research was supported in part by NSF Grant No. DMS-0209595. 


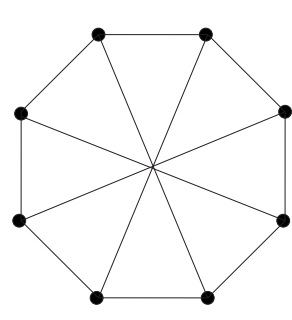

$V_{8}$

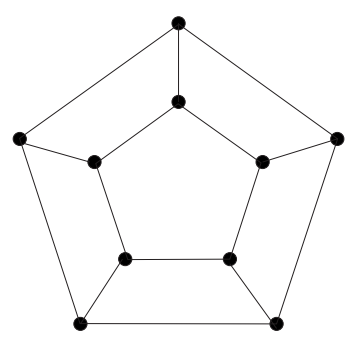

$C_{5} \times C_{2}$

Fig. 1. The graphs $V_{8}$ and $C_{5} \times C_{2}$.

Also note that we allow edges to have length 0 . It turns out that allowing edges of 0 length does not change which graphs are $d$-realizable.

In [BC1] Belk and Connelly give a complete classification of 3-realizable graphs, assuming that the graphs $V_{8}$ and $C_{5} \times C_{2}$ are 3-realizable (see Fig. 1 for the definitions of these two graphs). Assuming the following two theorems, they show that a graph is 3-realizable if and only if it does not contain $K_{5}$ or $K_{2,2,2}$ as a minor. In this paper the classification of 3-realizable graphs is completed with the following two theorems:

Theorem 1. The graph $V_{8}$ is 3-realizable.

Theorem 2. The graph $C_{5} \times C_{2}$ is 3-realizable.

In this paper the above two theorems will be proven using techniques from stress theory and tensegrities.

As was pointed out in [BC1], it should be possible to use techniques of real algebraic geometry to determine whether a specific graph is $d$-realizable. This would perhaps be a different way to solve the problem, but would take longer. Let $e=|E(G)|$ and $v=|V(G)|$, and suppose that we know that $G$ is $N$-realizable (for example, $N$ could be $v$ ). There is a polynomial function from $\mathbb{R}^{N v}$ to $\mathbb{R}^{e}$ which takes a realization in $\mathbb{E}^{N}$ and returns the length of each edge. The image of this polynomial function is a semi-algebraic set (a set defined by a finite list of polynomial inequalities). There is a similar polynomial function from $\mathbb{R}^{d v}$ to $\mathbb{R}^{e}$. The question of whether $G$ is $d$-realizable is then equivalent to the question of whether the two semi-algebraic sets are equal. This question can be solved, but the algorithm is exponential. One bound on the complexity is $(4 e)^{O\left(N d v^{2}\right)}$. See Chapter 13 of [BPR] for more information on determining whether two semi-algebraic sets are equal.

\section{Stress Theory}

The plan for proving Theorems 1 and 2 is to stretch realizations of $V_{8}$ and $C_{5} \times C_{2}$ so that two vertices are as far apart as possible without changing any edge lengths. Then we 
will argue that in most cases this stretching forces some of the vertices to span a threedimensional subspace, allowing the remaining vertices to rotate about the plane spanned by their neighbors and into the three-dimensional subspace. We say that a realization can be folded into $\mathbb{E}^{3}$, if it is possible to obtain a realization in $\mathbb{E}^{3}$ by repeatedly rotating different vertices about the plane spanned by the vertex's neighbors. Note that every vertex in $V_{8}$ and $C_{5} \times C_{2}$ has degree 3 , so the affine span of a vertex's neighbors has dimension 2 or less. The main tool used in this argument is stresses and tensegrities, as described in $[\mathrm{C}]$.

Definition 1. A tensegrity, denoted $G(\mathbf{p})$ is a configuration $\mathbf{p}=\left(\mathbf{p}_{\mathbf{1}}, \ldots, \mathbf{p}_{\mathbf{n}}\right)$ and a graph $G$, where each edge of the graph is labeled as a cable, strut, or bar, and where each vertex is labeled as being pinned or unpinned.

The idea is that cables are allowed to decrease in length (or stay the same length), but not to increase in length. Struts are allowed to increase in length (or stay the same length), but not to decrease in length. Bars are forced to remain the same length. The tensegrities that we are concerned with have bars (the edges of $V_{8}$ and $C_{5} \times C_{2}$, since we want these lengths to remain the same) and one strut (for the stretching). When drawing a tensegrity, we denote bars by a single line between vertices, struts by a double line between vertices, and cables by a dotted line between vertices.

Pinned vertices are forced to remain where they are. There is only one case where we consider a tensegrity with pinned vertices. Unless explicitly stated otherwise, vertices are assumed to be unpinned.

Definition 2. Fix a graph $G$. Let $\mathbf{p}$ and $\mathbf{q}$ be two configurations of $G$. If $G(\mathbf{q})$ satisfies the cable, strut, and bar conditions of $G(\mathbf{p})$, then we say that $G(\mathbf{p})$ dominates $G(\mathbf{q})$, which we denote by $G(\mathbf{p}) \succeq G(\mathbf{q})$. More precisely, $G(\mathbf{p}) \succeq G(\mathbf{q})$ if for every pinned vertex $i, p_{i}=q_{i}$, and for every edge $\{i, j\}$,

$$
\begin{array}{ll}
\left|p_{i}-p_{j}\right| \geq\left|q_{i}-q_{j}\right| & \text { if }\{i, j\} \text { is a cable, } \\
\left|p_{i}-p_{j}\right|=\left|q_{i}-q_{j}\right| & \text { if }\{i, j\} \text { is a bar, } \\
\left|p_{i}-p_{j}\right| \leq\left|q_{i}-q_{j}\right| & \text { if }\{i, j\} \text { is a strut. }
\end{array}
$$

For the tensegrities that we are interested in, $G$ is the graph $V_{8}$ or $C_{5} \times C_{2}$ with the edges labeled as bars and with an additional edge (labeled a strut) between two of the vertices, say $i$ and $j$. Suppose $\mathbf{p}$ and $\mathbf{q}$ are both configurations of $G$. Then $G(\mathbf{q})$ dominates $G(\mathbf{p})$ if the edge lengths of $V_{8}$ or $C_{5} \times C_{2}$ are the same in both $\mathbf{p}$ and $\mathbf{q}$ and the distance between $i$ and $j$ in $\mathbf{q}$ is either greater than or equal to the distance in $\mathbf{p}$.

Definition 3. An equilibrium stress for $G(\mathbf{p})$ is an assignment of real numbers $\omega_{i j}=\omega_{j i}$ to each edge $\{i, j\} \in E(G)$ such that for each unpinned vertex $i$ of $G$, $\sum_{j:\{i, j\} \in E(G)} \omega_{i j}\left(p_{i}-p_{j}\right)=0$. 


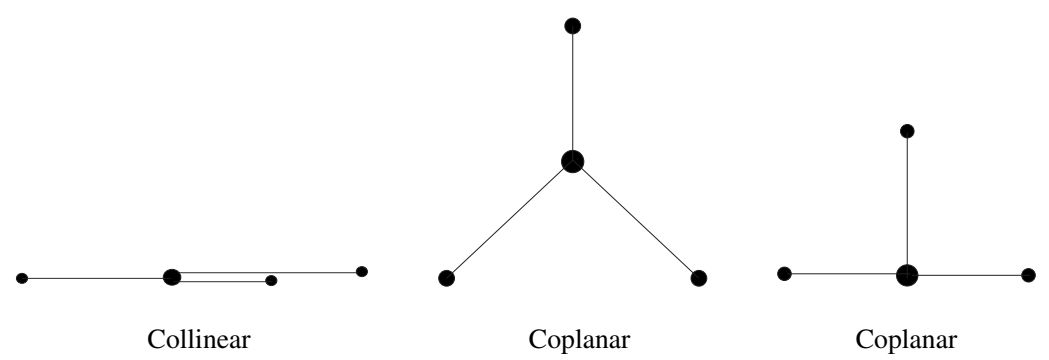

Fig. 2. The possible configurations for a degree 3 vertex with at least two adjacent non-zero stresses.

We denote a stress by a vector $\omega$, where the components of the vector range over the edges of $G$. Since every stress that we will be concerned with is an equilibrium stress, we often refer to an equilibrium stress as simply a stress.

The existence of an equilibrium stress is what we use to argue that certain vertices span low enough dimension. Note that if a vertex has degree 2 and both incident edges have a non-zero stress, then the vertex is collinear with its neighbors. Also, if a vertex has degree 3 and all three incident edges have a non-zero stress, then the vertex is coplanar or collinear with its neighbors. In fact if a vertex has degree 3 and at least two incident edges have a non-zero equilibrium stress, then the vertex is coplanar with its neighbors-if the stress is zero on one of the edges, then it is collinear with two of its neighbors and the third vertex creates the plane. Figure 2 shows the possible configurations for a degree 3 vertex with at least two adjacent non-zero stresses.

We need a way to show that a tensegrity has a non-zero equilibrium stress (non-zero on at least one edge). The condition we use is unyielding.

Definition 4. A tensegrity $G(\mathbf{p})$ is unyielding if any other configuration $\mathbf{q}$ with $G(\mathbf{p}) \succeq$ $G(\mathbf{q})$ has the same edge lengths as in $\mathbf{p}$.

Let $v$ denote the number of unpinned vertices of $G$, and let $e$ denote the number of edges in $G$ that are incident to at least one unpinned vertex. Consider the function $f: \mathbb{R}^{N v} \rightarrow \mathbb{R}^{e}$ which takes a realization in $\mathbb{E}^{N}$ and returns the square of the length of each edge in the realization: $f(\mathbf{p})=\left(\ldots,\left|p_{i}-p_{j}\right|^{2}, \ldots\right)$. Let $R(\mathbf{p})$ denote the derivative of this function at the realization $\mathbf{p}$. The matrix $R(\mathbf{p})$ is called the rigidity matrix. Then, for a given realization $\mathbf{p}, R(\mathbf{p})$ is a matrix with $e$ rows and $N v$ columns. The entry in row $\{i, j\}$ and in the $N$ columns corresponding to an unpinned vertex $i$ is the row vector $2\left(p_{i}-p_{j}\right)$. If $j$ is unpinned, then there is a corresponding $2\left(p_{j}-p_{i}\right)$ in row $\{i, j\}$ in the columns corresponding to vertex $j$. All other entries in the matrix are zero.

In the following example matrix, vertices $i$ and $j$ are unpinned, vertex $k$ is pinned, and $\{i, j\}$ and $\{i, k\}$ are both edges of $G$. Note that each row of the matrix corresponds to an edge, and $N$ columns of the matrix correspond to a vertex. In the example matrix, the rows corresponding to edges $\{i, j\}$ and $\{i, k\}$ and the columns corresponding to vertices $i$ and $j$ are shown. The entries in the columns corresponding to the pinned vertex $k$ are 
all zero:

$$
R(\mathbf{p})=\left(\begin{array}{ccccccc}
\multicolumn{9}{c}{\operatorname{vertex} i} & \operatorname{vertex} j & & \\
\vdots & & \vdots & \\
\cdots & 0 & 2\left(p_{i}-p_{j}\right) & \ldots & 2\left(p_{j}-p_{i}\right) & 0 & \ldots \\
\cdots & 0 & 2\left(p_{i}-p_{k}\right) & \ldots & 0 & 0 & \ldots \\
& \vdots & & \vdots &
\end{array}\right) \text { edge }\{i, j\}
$$

Note that if $\omega$ is in the cokernel of $R(\mathbf{p})$, then $\omega$ is an equilibrium stress for $G(\mathbf{p})$.

Theorem 3. If $G(\mathbf{p})$ is an unyielding tensegrity with exactly one strut or cable, then $G(\mathbf{p})$ has an equilibrium stress that is non-zero on at least one edge.

Proof. Suppose that the only equilibrium stress on $G(\mathbf{p})$ is zero on all edges, meaning that the cokernel of $R(\mathbf{p})$ is trivial. Then $R(\mathbf{p})$ is a surjective linear transformation. Thus, there exists an open neighborhood $U$ about $\mathbf{p} \in \mathbb{R}^{N v}$ and an open neighborhood $V$ about $f$ (p) such that $f$ maps $U$ onto $V$ (see the Rank Theorem in [R]). Thus, there exists a realization $\mathbf{q}$ such that $\mathbf{p}$ and $\mathbf{q}$ have the same bar lengths but $\mathbf{q}$ has a longer strut length or a shorter cable length. Then $G(\mathbf{p})$ is not unyielding. We are done by contrapositive.

The non-zero equilibrium stress that is obtained by the theorem is not necessarily non-zero on the strut or cable. Figure 3 shows an example of an unyielding tensegrity where the equilibrium stress is zero on the cable.

The following lemma gives us a way to obtain an unyielding tensegrity.

Lemma 1. Let $G(\mathbf{p})$ be a tensegrity with exactly one strut or cable and suppose that $G$ is connected and remains connected if the strut or cable is removed. Then there exists a configuration $\mathbf{q}$ with $G(\mathbf{p}) \succeq G(\mathbf{q})$ and $G(\mathbf{q})$ unyielding.

Proof. Let $v=|V(G)|$, and let $i$ and $j$ be the two vertices connected by the strut or cable. Note that every configuration of $G$ spans at most $v-1$ dimensions; thus, every configuration of $G$ exists in $\mathbb{R}^{v-1}$. Let $f: \mathbb{R}^{v \cdot(v-1)} \rightarrow \mathbb{R}^{1}$ be the function that takes a

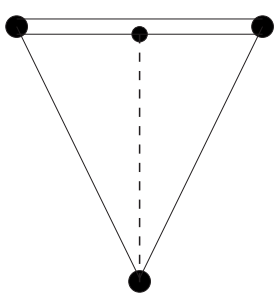

Fig. 3. This tensegrity is unyielding, and has a non-zero equilibrium stress by Theorem 3 . The equilibrium stress is non-zero on the degenerate triangle. It is zero on the cable. 
configuration in $R^{v-1}$ and returns the distance between vertices $i$ and $j$ (the strut or cable length). Consider the set of configurations that have the same bar lengths as in $G(\mathbf{p})$ and that have at least one of the vertices pinned (if none of the original vertices in $G$ is pinned, then pin vertex 1). Note that this set is compact: since $G$ with the strut or cable removed is connected, every vertex has a maximum distance it can be from the pinned vertex. Thus, $f$ attains a maximum on this set. This maximum occurs at an unyielding configuration $G(\mathbf{q})$, since there is no configuration with the same edge lengths and a longer strut length (or shorter cable length).

For more information on tensegrities and stress theory, see [C], [CW], and [RW].

\section{3-Realizability of $V_{8}$}

Let $\mathbf{p}$ be a realization of $V_{8}$. Our goal is to find a realization of $\mathbf{p}$ in $\mathbb{E}^{3}$ with the same edge lengths.

Consider the tensegrity with configuration $\mathbf{p}$, vertices $\{1,2,3, \ldots, 8\}$, bars the edges of $V_{8}$, and one strut incident to two vertices in $V_{8}$ that are three edges apart along the outer cycle (say vertices 1 and 4, as in Fig. 4). Call the resulting graph $G$. We use $V_{8}$ when we want to refer to just the graph $V_{8}$, and we use $G$ when we want to refer to $V_{8}$ plus a strut.

By Theorem 3 and Lemma 1, if $\mathbf{p}$ is a realization of $V_{8}$ then there is a realization $\mathbf{q}$ of $V_{8}$ with the same edge lengths as $\mathbf{p}$ where the tensegrity $G(\mathbf{q})$ has a non-zero equilibrium stress. We will show that this means that the realization can be folded into $\mathbb{E}^{3}$. First, we describe some situations where the realization of $V_{8}$ can be folded into $\mathbb{E}^{3}$. Then we show that if there is a non-zero stress on the tensegrity, then one of these situations is occurring. In the following lemma we are considering realizations of the graph $V_{8}$, not realizations of the tensegrity.

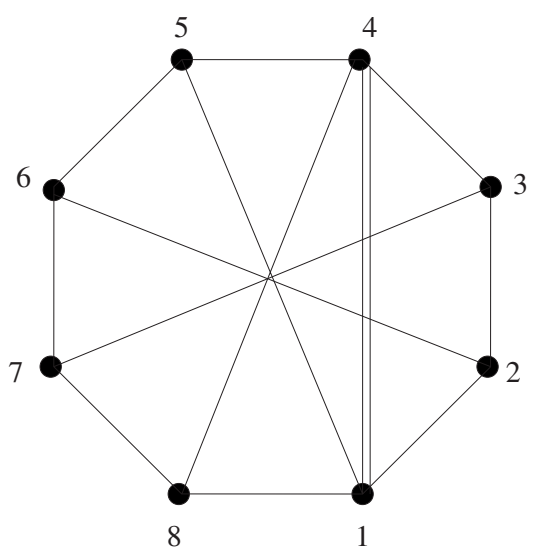

Fig. 4. The tensegrity $V_{8}$ with a strut. 
Lemma 2. If a realization $\mathbf{p}$ of $V_{8}$ has any of the following situations, then it can be folded into $\mathbb{E}^{3}$ :

1. Four vertices are collinear;

2. three vertices, not consecutive on the outer cycle, are collinear; or

3. each vertex in the set $\{3,6,7,8\}$ (or an isomorphic set) lies in the same plane or line as its neighbor.

Proof. If vertices $1,2,4,5$, and 7 are in $\mathbb{E}^{3}$, then each remaining vertex can be rotated about the plane spanned by its neighbors into $\mathbb{E}^{3}$. This works for any five vertices such that none of the remaining three vertices is adjacent. Up to symmetry, the vertices given are the only way to do this. If four vertices are collinear or if three non-consecutive vertices are collinear, then there are five such vertices that span a three-dimensional subspace. Thus, situations 2 and 2 can be folded into $\mathbb{E}^{3}$.

The proof of situation 2 is slightly more involved. We start with four vertices assumed to be in $\mathbb{E}^{3}$ and, one by one, show that the remaining vertices must also be in $\mathbb{E}^{3}$. The main plan to show that a vertex is in $\mathbb{E}^{3}$ is the following: suppose a vertex $i$ lies in a plane with its neighbors and suppose that vertex $i$ and two of its neighbors are known to be in $\mathbb{E}^{3}$, then either the third vertex is in $\mathbb{E}^{3}$ or vertex $i$ is collinear with its other two neighbors. In the cases below, if vertex $i$ is collinear with its other two neighbors, then situation 2 occurs. If vertex $i$ is collinear with its neighbors rather than coplanar and vertex $i$ and two of its neighbors are in $\mathbb{E}^{3}$, then the third vertex is also in $\mathbb{E}^{3}$. Because this case works the same way as the coplanar case, we just use the coplanar case in the argument.

Vertices 2, 3, 6, and 7 span at most a three-dimensional space. We assume that $p_{2}, p_{3}, p_{6}, p_{7} \in \mathbb{E}^{3}$. If they span fewer dimensions, the same argument will still workeach new vertex considered will either be in the same low-dimensional space or will make the space into $\mathbb{E}^{2}$ or $\mathbb{E}^{3}$.

Vertex 6 lies in the same plane as its neighbors, vertices 2, 5, and 7. This plane includes vertices 2, 6, and 7 all of which are in $\mathbb{E}^{3}$. If 2,6 , and 7 do not span all of the plane, then they lie on a line and the entire realization can fold into $\mathbb{E}^{3}$ by situation 2 . Otherwise, the entire plane is in $\mathbb{E}^{3}$, so vertex 5 is also in $\mathbb{E}^{3}$.

By a similar argument, vertex 7 lying in a plane with its neighbors forces vertex 8 to be in $\mathbb{E}^{3}$ (or else situation 2 applies), and vertex 3 lying in a plane with its neighbors forces vertex 4 to be in $\mathbb{E}^{3}$ (or else situation 2 applies). Now we have $p_{2}, p_{3}, p_{4}, p_{5}, p_{6}, p_{7}, p_{8} \in$ $\mathbb{E}^{3}$.

The only vertex not yet shown to be in $\mathbb{E}^{3}$ is vertex 1 . Vertex 8 lies in the same plane as its neighbors, vertices 1,4 , and 7 . Since 4,7 , and 8 are all in $\mathbb{E}^{3}$, vertex 1 must also lie in $\mathbb{E}^{3}$ (or else situation 2 applies).

Thus, all of the vertices are in $\mathbb{E}^{3}$ or can be folded into $\mathbb{E}^{3}$.

Theorem 1. $V_{8}$ is 3-realizable.

Proof. Let $\mathbf{p}$ be a realization of $V_{8}$. Consider $G(\mathbf{p})$ the tensegrity with configuration $\mathbf{p}$, bars for the edges of $V_{8}$, and one strut as in Fig. 4. By Lemma 1 and Theorem 3, this tensegrity has an unyielding realization $\mathbf{q}$ such that $G(\mathbf{q})$ has a non-zero stress. We will 
show that a non-zero stress on the tensegrity implies that we are in one of the above situations of Lemma 2, which means that we can fold $V_{8}$ into $\mathbb{E}^{3}$.

We call a vertex a zero vertex if all of the incident edges have a stress of zero, and we call a vertex a non-zero vertex if at least one of the incident edges has a non-zero stress. We assume that the strut is incident to vertices 1 and 4 (as in Fig. 4).

Case 1: Vertex 5, 6, 7, or 8 is a zero vertex. Suppose vertex 5 is a zero vertex. Vertex 6 is adjacent to 5 , so the edge $\{5,6\}$ has a stress of zero. Then either vertex 6 has exactly two incident edges with non-zero stress or all edges incident to vertex 6 have zero stress, making 6 a zero vertex. If vertex 6 has exactly two incident edges with non-zero stress, then vertices 2, 6, and 7 are collinear, which implies situation 2 , meaning $V_{8}$ can fold into $\mathbb{E}^{3}$. Thus, 6 must be a zero vertex. Similarly, if any of $5,6,7$, or 8 is a zero vertex, then all four vertices must be zero vertices (or we have situation 2). In this case the non-zero stress lies entirely on vertices $1,2,3$, and 4 . The only way for this subgraph to have a non-zero stress is for all four of these vertices to be collinear (situation 2). Thus, all possibilities in this case can be folded into $\mathbb{E}^{3}$.

Case 2: Vertex 2 or 3 is a zero vertex. Then, by the same argument as above, either 2 and 3 are both zero vertices, or situation 2 occurs. Thus, we are concerned with the case where the non-zero stress lies entirely on vertices in the set $\{1,4,5,6,7,8\}$. Since 2 is zero, either 6 is a zero vertex (see case 1) or vertices 5, 6, and 7 are collinear. Similarly, vertex 3 being a zero vertex implies either case 1 or that vertices 6,7 , and 8 are collinear. Thus, either case 1 occurs, or vertices 5-8 are all collinear (situation 2).

Case 3: None of vertices 2, 3, 5, 6, 7, or 8 is a zero vertex. Then each of these vertices must lie in the same plane as their neighbors, so we have situation 2.

In each of the possible cases, we see that $V_{8}$ can be folded into $\mathbb{E}^{3}$. Thus, $V_{8}$ is 3-realizable.

\section{3-Realizability of $C_{5} \times C_{2}$}

Consider the tensegrity with vertices $\{1,2,3, \ldots, 10\}$, bars for the edges of the graph $C_{5} \times C_{2}$, and a strut between vertices 3 and 8 , as shown in Fig. 5 . The strut is chosen so that the smallest cycle in the graph of the tensegrity has length 4 . Call the resulting
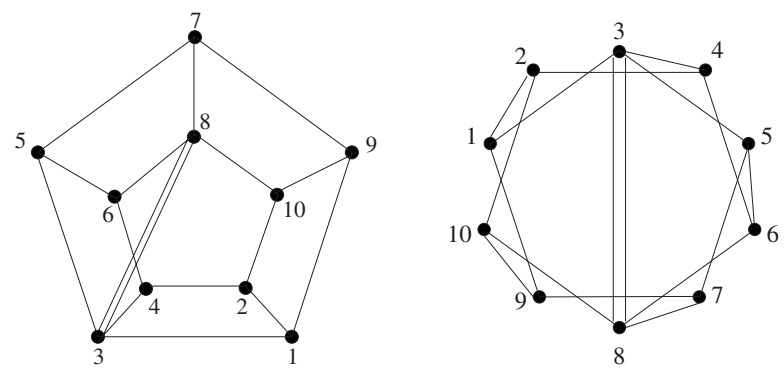

Fig. 5. Two drawings of $C_{5} \times C_{2}$ with an added strut. 
graph $G$. We use $C_{5} \times C_{2}$ when we want to refer to just the graph $C_{5} \times C_{2}$, and we use $G$ when we want to refer to $C_{5} \times C_{2}$ plus a strut.

By Lemma 1 and Theorem 3, this tensegrity must have an unyielding configuration with a non-zero stress. We will show that from this stressed position one of the following must occur:

- $C_{5} \times C_{2}$ can be folded into $\mathbb{E}^{3}$,

- we can pin the stressed vertices and add a strut between two other vertices, resulting in another stressed configuration that can be folded into $\mathbb{E}^{3}$, or

- there is a sequence of realizations of $C_{5} \times C_{2}$ that can be realized in $\mathbb{E}^{3}$ converging to the unyielding configuration, implying that the unyielding configuration has a realization in $\mathbb{E}^{3}$. This possibility will only be used for the case where the vertices of a cycle of $C_{5} \times C_{2}$ are collinear.

The stress is non-zero on a subgraph of $C_{5} \times C_{2}$. Our plan will be to examine all such possible subgraphs. At first, this seems like we want to examine all $2^{16}$ subgraphs of $C_{5} \times C_{2}$, but a vertex with degree 1 cannot have a non-zero stress on that edge. Thus, we only need to consider those subgraphs where no vertex has degree 1 . A computer computation using Maple [M] reveals that there are 1665 such subgraphs. Through additional simplifications, we reduce the number of graphs that we need to look at even further.

We do not rely on computer computation, but rather work through all cases, ignoring situations that create a degree 1 vertex. However, it is comforting to know that further computer computation arrives at exactly the same cases. We break the argument into the following cases:

1. The subgraph contains a cycle that is forced collinear by the stress (for example, if the subgraph is a cycle, then it is forced collinear by the stress).

2. We remove one edge from $G$.

3. We remove two edges from $G$ without creating a vertex of degree 1 .

4. We remove one vertex from $G$ (and possibly remove some edges).

5. We remove two vertices from $G$ (and possibly remove some edges).

6. We remove three vertices from $G$ (and possibly remove some edges).

7. We remove four vertices from $G$ (and possibly remove some edges).

A subgraph with more than four vertices removed must either contain a cycle that is forced collinear by the stress (case 1) or have a vertex with degree 1 .

In each case we get a list of possible subgraphs with a stress that is non-zero on each edge. Then we argue that the stressed graph must span an appropriate dimension, so that $C_{5} \times C_{2}$ can fold into $\mathbb{E}^{3}$. In one case (Case 7 above), we get a graph that is not of the appropriate dimension. In that case we pin the stressed vertices and add a second strut, resulting in a second stress. This second stress will force $C_{5} \times C_{2}$ to be in the appropriate dimension so that we can fold it into $\mathbb{E}^{3}$.

If a vertex in the subgraph has degree 2 , then it must be collinear with its neighbors. Thus, for the purpose of determining the maximum dimension spanned by the stressed graph, we can replace the degree 2 vertex by an edge connecting its two neighbors. In fact, the tensegrity that results from replacing a degree 2 vertex with an edge between the neighbors has an equilibrium stress on it that comes from the original stress. 
Lemma 3. Let $G(\mathbf{p})$ be a tensegrity, with vertex $i$ having degree 2. Let the neighbors of vertex $i$ be vertices $j$ and $k$. Let $H$ be the graph obtained from $G$ by removing vertex $i$ and its incident edges and adding the edge $\{j, k\}$ (if the edge does not already exist). Let the configuration $\mathbf{q}$ be the same as $\mathbf{p}$ but not including vertex $i$. If $G(\mathbf{p})$ has an equilibrium stress, then $H(\mathbf{q})$ has an equilibrium stress.

Proof. Let $\omega$ denote the equilibrium stress on $G(\mathbf{p})$; we create the equilibrium stress $\alpha$ on $H(\mathbf{q})$. For most edges of $H$, we use the equilibrium stress from $G(\mathbf{p})$. For the edge $\{j, k\}$, the stress is

$$
\alpha_{j k}=\omega_{i j} \frac{\left\|\mathbf{p}_{i}-\mathbf{p}_{j}\right\|}{\left\|\mathbf{p}_{j}-\mathbf{p}_{k}\right\|}+\omega_{j k} .
$$

Since $\omega$ is an equilibrium stress (in particular, it satisfies the equilibrium condition at vertex $\left.i-\omega_{i j}\left(\mathbf{p}_{i}-\mathbf{p}_{j}\right)+\omega_{i k}\left(\mathbf{p}_{i}-\mathbf{p}_{k}\right)=0\right)$, this is equivalent to

$$
\alpha_{j k}=\omega_{i k} \frac{\left\|\mathbf{p}_{i}-\mathbf{p}_{k}\right\|}{\left\|\mathbf{p}_{j}-\mathbf{p}_{k}\right\|}+\omega_{j k} .
$$

This stress $\alpha$ satisfies the equilibrium condition at vertices $j$ and $k$.

When we examine the subgraphs in each of the cases, we often perform this operation (removing degree 2 vertices) without commenting on it.

The following two lemmas will be helpful in the case checking. This first lemma will be helpful in Case 1, as it allows us to deal only with cycles that include the strut.

Lemma 4. If there are realizations of $C_{5} \times C_{2}$ that cannot be realized in $\mathbb{E}^{3}$, then there exists such a realization where the lengths of the edges along a cycle cannot be added and subtracted to give zero.

Proof. Let $v=\left|V\left(C_{5} \times C_{2}\right)\right|$ and let $e=\left|E\left(C_{5} \times C_{2}\right)\right|$. Consider the function $f: \mathbb{R}^{3 v} \rightarrow \mathbb{R}^{e}$ that takes a realization of $C_{5} \times C_{2}$ in $\mathbb{E}^{3}$ and returns the lengths of each edge. The image of a closed ball of radius $M$ under this $f$ is a compact set, since $f$ is a continuous function. Thus, the set of edge lengths with no realization in $\mathbb{E}^{3}$ inside a closed ball of radius $M$ is an open set. Since every list of edge lengths with a realization in $\mathbb{E}^{3}$ has a realization inside a closed ball with a sufficiently large radius $M$, the set of edge lengths with no realization in $\mathbb{E}^{3}$ is open. Thus, given a list of edge lengths that cannot be realized in $\mathbb{E}^{3}$ where the lengths of the edges along one of the cycles can be added and subtracted to give zero, there must be a nearby list of edge lengths without this property that can also not be realized in $\mathbb{E}^{3}$.

The main point in the above proof is that the set of all realizations that cannot be realized in $\mathbb{E}^{3}$ is open. Similarly, we can assume that all edge lengths are non-zero. Alternatively, we could note that if an edge length is zero, then the actual graph being considered is a minor of $C_{5} \times C_{2}$, and therefore is 3-realizable according to [BC1].

Since we will be trying to bound the dimension of the span of certain stressed tensegrities, it will be helpful to have a condition on the number of vertices that bounds the span of the tensegrity. 
Lemma 5. Let $G(\mathbf{p})$ be a tensegrity with a stress that is non-zero on all edges. If $G$ has $n$ vertices, then the dimension of the span of the $n$ vertices is at most $n-2$. Additionally, if $G$ is not the complete graph on $n$ vertices, then the dimension of the span of the $n$ vertices is at most $n-3$.

Proof. The only tensegrity in $\mathbb{E}^{n-2}$ with $n$ vertices and a stress that is non-zero on all edges has $G=K_{n}$ (see [BC2]).

As an example of how some of the arguments for the various cases look, we do the case where the tensegrity $C_{5} \times C_{2}$ with an added strut has a stress that is non-zero on all the edges.

Lemma 6. Consider the tensegrity $G(\mathbf{p})$ shown in Fig. 5. If there is a stress that is non-zero on every edge of this tensegrity, then the configuration $\mathbf{p}$ lies in $\mathbb{E}^{3}$.

Proof. Every vertex that has degree 3 must be either coplanar or collinear with its neighbors, because there is a non-zero stress on all three incident edges. Also, if a degree 3 vertex is collinear with two of its neighbors, then it must be collinear with the third neighbor, since there must be a non-zero stress on the third edge. The four vertices 1 , 2,9 , and 10 span at most three dimensions. For simplicity, we call this three-dimensional space $\mathbb{E}^{3}$. (If these four vertices span two dimensions or fewer, the entire tensegrity will span fewer than three dimensions.)

The plan is similar to the plan for situation 2 in Lemma 2. We will show that a vertex is in $\mathbb{E}^{3}$ using the following argument: if vertex $i$ lies in a plane or a line with its neighbors and vertex $i$ and two of its neighbors are known to be in $\mathbb{E}^{3}$ and to span the plane or line, then the third vertex is also in $\mathbb{E}^{3}$.

Vertex 1 lies in a plane (or a line) with its neighbors, vertices 2, 3, and 9. This plane (or line) is spanned by vertices 1,2 , and 9 , all of which are in $\mathbb{E}^{3}$. Thus, vertex 3 is also in $\mathbb{E}^{3}$.

Similarly, vertex 2 lies in a plane (or line) with its neighbors, so vertex 4 is in $\mathbb{E}^{3}$. Vertex 9 lies in a plane (or line) with its neighbors, so vertex 7 is in $\mathbb{E}^{3}$. Vertex 10 lies in a plane (or line) with its neighbors, so vertex 8 is in $\mathbb{E}^{3}$. We now have all of $1,2,3,4,7$, 8,9 , and 10 in $\mathbb{E}^{3}$.

Vertex 7 lies in a plane (or line) with its neighbors, vertices 5,8 , and 9. This plane (or line) is spanned by vertices 7,8 , and 9 , all of which are in $\mathbb{E}^{3}$. Thus, vertex 5 is also in $\mathbb{E}^{3}$.

Vertex 5 lies in a plane (or line) with its neighbors, vertices 3, 6, and 7. This plane (or line) is spanned by vertices 3,5 , and 7 , which are all in $\mathbb{E}^{3}$. Thus, vertex 6 is also in $\mathbb{E}^{3}$.

Therefore, if there is a non-zero stress on every edge of this tensegrity, all vertices are in $\mathbb{E}^{3}$.

In the above proof we started with four vertices, which could span at most three dimensions. Then we used vertices known to be in $\mathbb{E}^{3}$ that were coplanar with their neighbors so that we could argue that another vertex was also in $\mathbb{E}^{3}$. We were able to show that all vertices were in $\mathbb{E}^{3}$. Similar arguments will be helpful in many of the cases. 
To simplify the later proofs, we use the notation $\langle a\rangle$ to mean the affine span of the vertex $a$ and the neighbors of $a$. We use the notation $\langle a\rangle \Rightarrow b$ to mean that

- $b$ is a neighbor of $a$,

- $\langle a\rangle$ is a line or a plane (that is, $a$ is coplanar or collinear with its neighbors),

- $a$ and its neighbors other than $b$ are known to be in $\mathbb{E}^{3}$,

- and because of this $b$ is also in $\mathbb{E}^{3}$.

A proof that a tensegrity is in $\mathbb{E}^{3}$ will consist of four starting vertices assumed to be in $E^{3}$ and multiple implications of the form $\langle a\rangle \Rightarrow b$. For the proof to work, we need all edges involved to be stressed and every vertex to be either a starting vertex or eventually to be a $b$ implied by some $\langle a\rangle$.

If we wish to show that a stressed graph is in $\mathbb{E}^{2}$, then we use the same notation, with $\langle a\rangle \Rightarrow b$ meaning

- $b$ is a neighbor of $a$,

- $\langle a\rangle$ is a line,

- $a$ and its neighbor other than $b$ are in $\mathbb{E}^{2}$ and span the line,

- and because of this, $b$ is in $\mathbb{E}^{2}$.

It should be clear from the context whether we are showing that the graph is in $\mathbb{E}^{3}$ or $\mathbb{E}^{2}$.

\subsection{Two Special Cases}

We start by examining two cases which are different from most of the other cases. In the first case there is a cycle of length 4 that includes the strut and is collinear. In the second case there is a stress that is zero on all edges incident to vertices 1, 2, 9, and 10, and non-zero on all the other edges.

Before we begin the first of these cases, we need a lemma that will allow us to apply $Y-\Delta$ transformations. A $Y-\Delta$ transformation is an operation applied to a graph that removes a vertex $i$ with degree 3 , and adds edges between all pairs of vertices adjacent to vertex $i$. The next lemma allows us to perform $Y-\Delta$ operations when trying to realize a graph in $\mathbb{E}^{3}$. If the resulting graph is 3 -realizable, then so is the original graph. However, if the resulting graph is not 3 -realizable, we do not know whether the original graph is 3-realizable.

Lemma 7. Let $G$ be a graph and let $\mathbf{p}=\left(\mathbf{p}_{\mathbf{1}}, \ldots, \mathbf{p}_{\mathbf{n}}\right)$ be a realization of the graph. Let $H$ and $\mathbf{q}=\left(p_{1}, \ldots, p_{i-1}, p_{i+1}, \ldots, p_{n}\right)$ be the graph and realization obtained by performing a $Y-\Delta$ transformation on a vertex $i$ of $G$. If there is a realization of $H$ in $\mathbb{E}^{3}$ with the same edge lengths as in $\mathbf{q}$, then there is a realization of $G$ in $\mathbb{E}^{3}$ with the same edge lengths as in $\mathbf{p .}$

Proof. We can construct the realization of $G$ by realizing $H$, and then folding the vertex $i$ into $\mathbb{E}^{3}$ along the plane formed by the vertices adjacent to $i$.

We are now ready to prove the first of the two cases. 


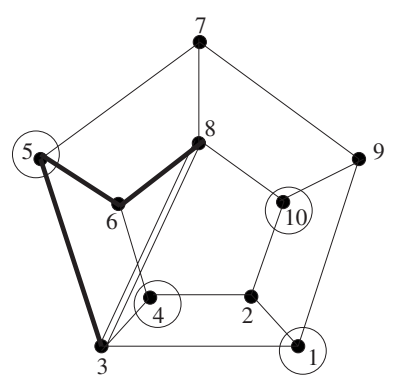

(a)

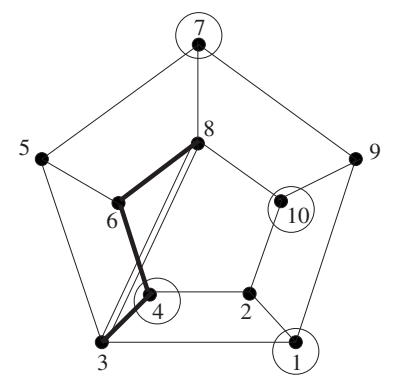

(b)

Fig. 6. The bold edges are collinear. The results of $Y-\Delta$ transformations on the circled vertices are the graphs in Fig. 7.

Lemma 8. Let $G(\mathbf{p})$ be an unyielding configuration of the tensegrity $C_{5} \times C_{2}$ plus a strut as shown in Fig. 5. Suppose that there is a cycle of length 4 which includes the strut that is collinear. Then there is a realization of $C_{5} \times C_{2}$ in $\mathbb{E}^{3}$ with the given edge lengths.

Proof. We assume that the three edges shown in bold in Fig. 6(a) or 6(b) are collinear. We can perform $Y-\Delta$ transformations on the indicated vertices to get the graph shown in Fig. 7 (here we are just concerned with the graph $C_{5} \times C_{2}$ not the tensegrity $C_{5} \times C_{2}$ plus a strut). Vertices 3,6 , and 8 are collinear. By Lemma 7 , if the resulting configuration can be realized in $\mathbb{E}^{3}$, the original configuration can also be realized in $\mathbb{E}^{3}$.

We would like to show that any realization of the graph in Fig. 7 with vertices 3, 6, and 8 collinear can be folded into $\mathbb{E}^{3}$. Since vertices 3,6 , and 8 are collinear, we can assume that vertices $3,6,8,2$, and 9 are all in $\mathbb{E}^{3}$. We can rotate the remaining vertex, vertex 7 or 5 , about the plane spanned by vertices $3,6,8$, and 9 and into $\mathbb{E}^{3}$.

Lemma 9. Let $G(\mathbf{p})$ be an unyielding configuration of the tensegrity $C_{5} \times C_{2}$ plus a strut as shown in Fig. 5. Suppose that there is a stress that is zero on all edges incident to vertices 1, 2, 9, and 10 and non-zero on all other edges. Then there exists a realization in $\mathbb{E}^{3}$ with the same edge lengths.

Proof. We have the situation shown in Fig. 8. Vertices $3,4,5,6,7$, and 8 are all stressed, and vertices 1, 2, 9, and 10 are not stressed. The stress on vertices $3,4,5,6,7$, and 8 forces

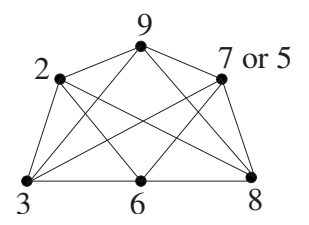

Fig. 7. This graph is the result of applying $Y-\Delta$ transformations to the graphs in Fig. 6. The vertex labeled "7 or 5 " is vertex 7 when $Y-\Delta$ transformations are applied to Fig. 6(a), and vertex 5 when applied to Fig. 6(b). 


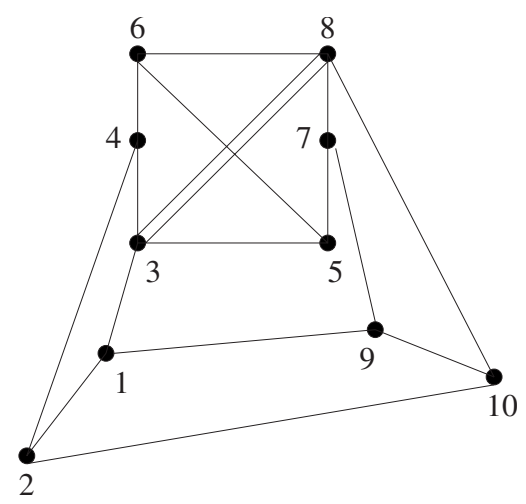

Fig. 8. There is a non-zero stress on the edges incident to vertices $3,4,5,6,7$, and 8 , and a zero stress on the edges incident to vertices $1,2,9$, and 10 . The stressed vertices are all coplanar.

those vertices to be coplanar. It is not clear how to move the remaining vertices into a three-dimensional subspace containing this plane (in later examples, we will be able to rotate vertices about the plane spanned by their neighbors and into a three-dimensional subspace, but that does not work in this case).

Consider pinning vertices $3,4,5,6,7$, and 8 (the stressed vertices) and adding a strut between vertices 4 and 9 (see Fig. 9). By Lemma 1 and by Theorem 3, the resulting tensegrity must have an unyielding configuration with a non-zero stress. Now we just need to show that this new stress forces the tensegrity into an appropriate dimension.

We consider all possibilities for this new non-zero stress.

There is only one way to have a non-zero stress at only one unpinned vertex-edges $\{4,9\}$ and $\{9,7\}$ could have a non-zero stress, with all other edges having a stress of zero. Then vertex 9 lies in the same plane as vertices $3-8$. Vertex 2 forms $\mathbb{E}^{3}$, and vertices 1 and 10 can fold into $\mathbb{E}^{3}$.

If there are exactly two unpinned vertices with non-zero stress on some of the incident edges, then the two vertices must lie coplanar with pinned vertices. One of the remaining vertices forms $\mathbb{E}^{3}$, and the final vertex can be folded into $\mathbb{E}^{3}$.

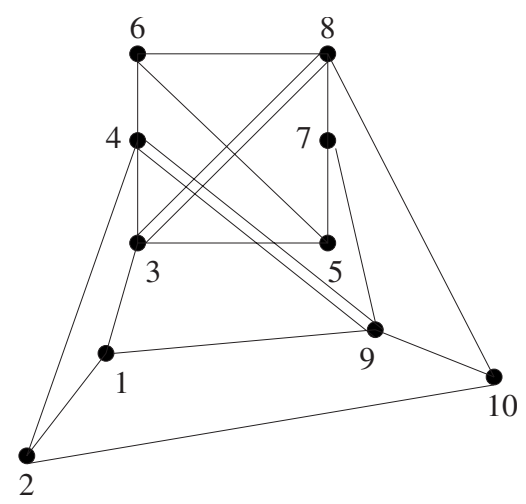

Fig. 9. Adding a strut to Fig. 8. 
Consider the case where three unpinned vertices have non-zero stresses on incident edges. Up to symmetry, there are two possible cases:

1. The stressed vertices are 1,2 , and 10 . In this case vertices 1 and 10 have only two adjacent non-zero stresses, so they are collinear with their neighbors.

2. The stressed vertices are 1,2, and 9. In this case vertex 2 must have only two adjacent non-zero stresses, so it is collinear with its neighbors. Also, vertex 1 may have two or three adjacent non-zero stresses, so it may be coplanar with its neighbors or collinear with two of its neighbors.

In case 1 and in the second possibility for case 2, there are two vertices that are forced collinear with their neighbors and there is a third stressed vertex ( 2 or 9$)$. Then the pinned vertices plus the third vertex (vertex 2 or vertex 9 ) forms $\mathbb{E}^{3}$. The vertices forced collinear are in $\mathbb{E}^{3}$ since they lie collinear with vertices in $\mathbb{E}^{3}$. The final vertex (vertex 9 or 10) can be rotated into $\mathbb{E}^{3}$. In the first possibility for case 2 , one vertex is forced collinear, then a second vertex (vertex 1) is forced coplanar with its neighbors. This plane determined by vertices 1,2 , and 9 intersects the plane of the pinned vertices along edge $\{3,4\}$ and contains the third stressed vertex. Thus, all of the stressed vertices and the pinned vertices lie in $\mathbb{E}^{3}$. The remaining vertex can be folded into $\mathbb{E}^{3}$.

Now, consider the case where all four unpinned vertices are stressed, with each edge that connects the vertices 1,2 , and 10 to the pinned vertices having a non-zero stress. If all four vertices are stressed, these four vertices span at most a three-dimensional subspace. Each of these stressed vertices except for 9 is coplanar with its neighbors. Vertex 1 forces vertex 3 to be in the three-dimensional subspace, vertex 2 forces vertex 4 to be in the three-dimensional subspace, and vertex 10 forces vertex 8 to be in the three-dimensional subspace. If vertices 3,4 , and 8 span the plane of the pinned vertices, then all of the vertices are in $\mathbb{E}^{3}$. If vertices 3,4 , and 8 do not span the plane of the pinned vertices, then vertices $3,4,6$, and 8 all lie on a line. This case was dealt with in Lemma 8.

Finally, all four vertices could be stressed with a zero stress on some or all of the edges connecting vertices 1,2 , and 10 to the pinned vertices. In that case, the vertex with an adjacent zero stress, must be collinear with its other neighbors. Then vertices 1, 2, 9, and 10 span either a plane or a line. If they span a plane, then only one of the four edges connecting to the pinned vertices has a zero stress. Then two of the pinned vertices are in the same plane as $1,2,9$, and 10 , and $\mathbb{E}^{3}$ is formed by the two intersecting planes: the plane containing the four stressed vertices and the plane containing the four pinned vertices. If the stressed vertices form a line, then $\mathbb{E}^{3}$ is formed by the plane of pinned vertices and the line of stressed vertices.

\subsection{The Remaining Cases}

Theorem 4. The graph $C_{5} \times C_{2}$ is 3-realizable.

Proof. Let $\mathbf{p}$ be a realization of $C_{5} \times C_{2}$. Consider $G(\mathbf{p})$ the tensegrity with configuration $\mathbf{p}$, bars for the edges of $C_{5} \times C_{2}$, and one strut as in Fig. 5. By Lemma 1 and Theorem 3, this tensegrity must have an unyielding realization $\mathbf{q}$ with a non-zero stress. We consider 
all possible cases where this stress could be, and in each case show that there is a realization in $\mathbb{E}^{3}$ with the same edge lengths for the edges of $C_{5} \times C_{2}$.

In these cases we call an edge removed if it has zero stress and a vertex removed if all incident edges have zero stress. We will be considering the subgraph of $C_{5} \times C_{2}$ that does not contain these removed vertices and edges. The resulting graph is the graph that results from this subgraph by replacing degree 2 vertices with edges. By Lemma 3, it suffices to show that the resulting graph is in the appropriate dimension.

Case 1: A cycle of $G$ is collinear. Note that the graph $C_{5} \times C_{2}$ plus a strut contains no triangles, so the cycle must have at least four vertices. By Lemma 4 , we can assume that the lengths of the bars along a cycle cannot be added and subtracted to give zero. This allows us to ignore the case where the stress lies on a cycle of length 4 or 5 consisting only of bars. If the stress lies on a four cycle including the strut, then the configuration can be folded into $\mathbb{E}^{3}$ by Lemma 8 .

Now suppose that the stress lies on a cycle of length 5 , including the strut. Then the stress lies on the strut plus the bold edges of one of the graphs in Fig. 10. The five vertices along the cycle must lie on a line. The two circled vertices form $\mathbb{E}^{3}$. The remaining vertices can be folded into $\mathbb{E}^{3}$ since they are not adjacent.

Finally, suppose that the stress lies on a cycle of length 6 or more. Then of the four vertices not in the cycle, at least two of them must be not adjacent (since the graph contains no triangles). Thus, the six vertices lie on a line, one vertex forms a plane, one vertex forms $\mathbb{E}^{3}$, and the two non-adjacent vertices can both be folded into $\mathbb{E}^{3}$.

Case 2: Every edge except one has a non-zero stress. First note that the proof of Lemma 6 (which does the case where every edge has a non-zero stress) did not use the assumption
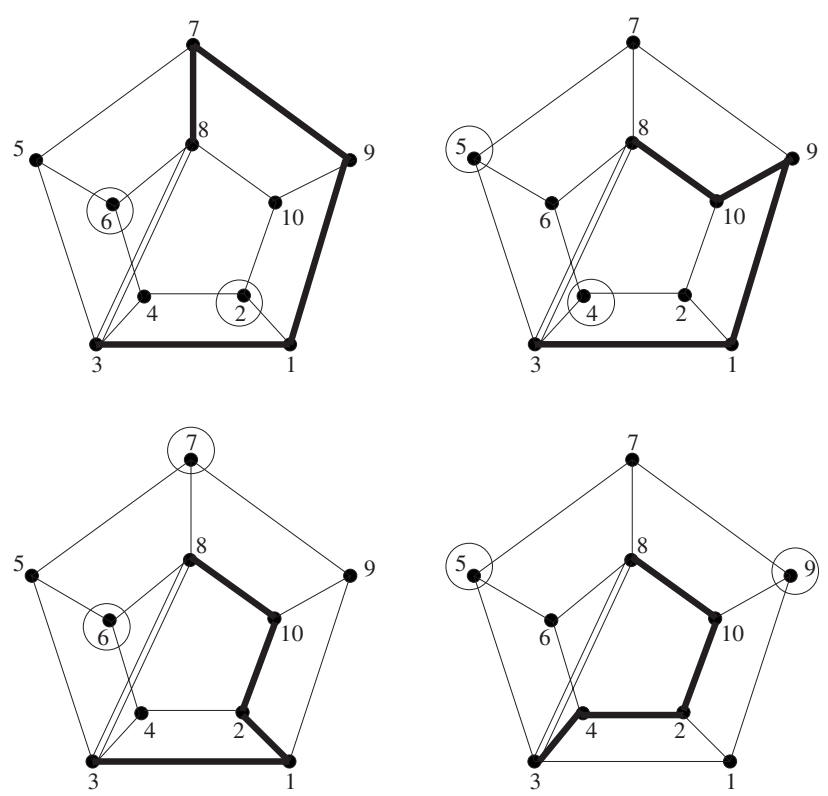

Fig. 10. The bold edges plus the strut form a cycle of length 5 . If this cycle of length 5 lies on a line, then the circled vertices form $\mathbb{E}^{3}$ and the remaining vertices can be folded into $\mathbb{E}^{3}$. 
that the strut had a non-zero stress on it. Thus, the proof of Lemma 6 does the case where every edge except the strut has a non-zero stress.

Using the second drawing of $G$ from Fig. 5, we see that there is a twofold symmetry (flip the graph around the horizontal line through the center of the drawing). Thus, up to symmetry and not including the strut, there are eight possible edges to have zero stress. From each subgraph, we replace the degree 2 vertices with an edge between its two neighbors (because this does not affect the maximum span of the stressed graph). The resulting graphs are shown in Fig. 11. The circled vertices will be used in proving that the stressed graphs span at most three dimensions.

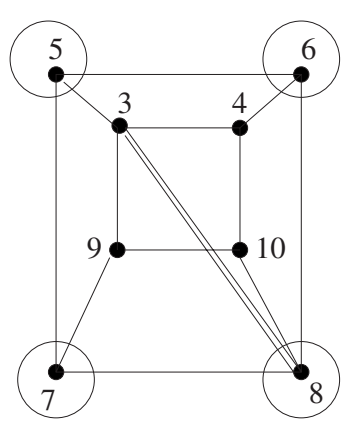

$\{1,2\}$

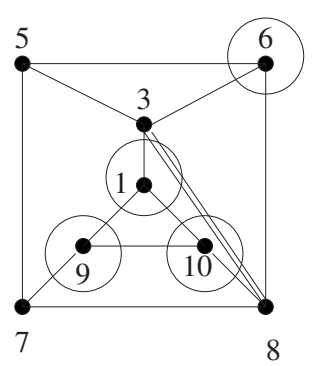

$\{2,4\}$

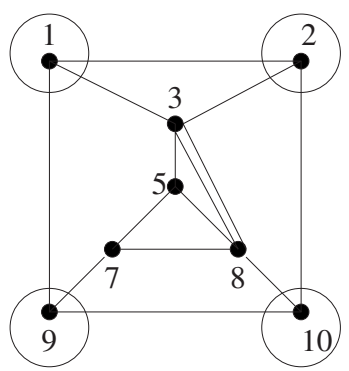

$\{4,6\}$

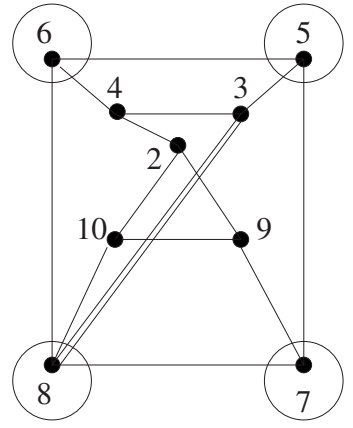

$\{1,3\}$

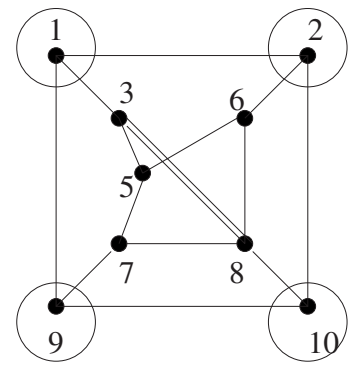

$\{3,4\}$

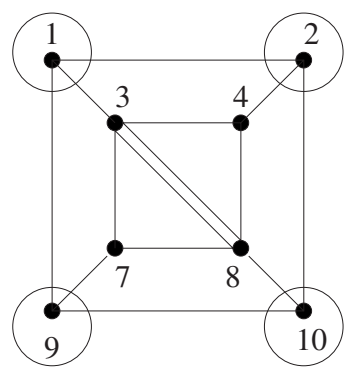

$\{5,6\}$

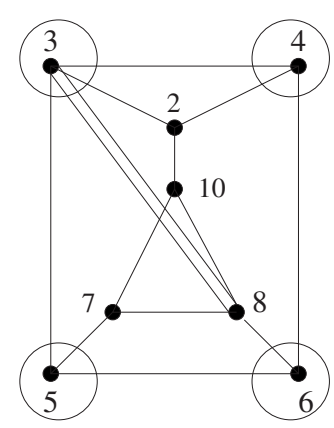

$\{1,9\}$

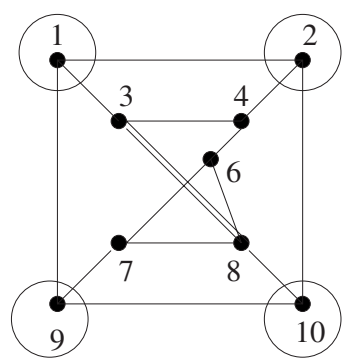

$\{3,5\}$

Fig. 11. The graphs resulting from removing one edge. 
Table 1. Each of the stressed graphs for removing one edge (see Fig. 11) spans at most three dimensions.

\begin{tabular}{lll}
\hline$\{1,2\}$ & $5,6,7,8 \in \mathbb{E}^{3}$ & $\langle 5\rangle \Rightarrow 3,\langle 6\rangle \Rightarrow 4,\langle 7\rangle \Rightarrow 9,\langle 9\rangle \Rightarrow 10$ \\
$\{1,3\}$ & $5,6,7,8 \in \mathbb{E}^{3}$ & $\langle 5\rangle \Rightarrow 3,\langle 6\rangle \Rightarrow 4,\langle 7\rangle \Rightarrow 9,\langle 4\rangle \Rightarrow 2,\langle 2\rangle \Rightarrow 10$ \\
$\{1,9\}$ & $3,4,5,6 \in \mathbb{E}^{3}$ & $\langle 4\rangle \Rightarrow 2,\langle 2\rangle \Rightarrow 10,\langle 5\rangle \Rightarrow 7,\langle 6\rangle \Rightarrow 8$ \\
$\{2,4\}$ & $1,6,9,10 \in \mathbb{E}^{3}$ & $\langle 1\rangle \Rightarrow 3,\langle 9\rangle \Rightarrow 7,\langle 10\rangle \Rightarrow 8,\langle 6\rangle \Rightarrow 5$ \\
$\{3,4\}$ & $1,2,9,10 \in \mathbb{E}^{3}$ & $\langle 1\rangle \Rightarrow 3,\langle 2\rangle \Rightarrow 6,\langle 9\rangle \Rightarrow 7,\langle 10\rangle \Rightarrow 8,\langle 7\rangle \Rightarrow 5$ \\
$\{3,5\}$ & $1,2,9,10 \in \mathbb{E}^{3}$ & $\langle 1\rangle \Rightarrow 3,\langle 2\rangle \Rightarrow 4,\langle 9\rangle \Rightarrow 7,\langle 10\rangle \Rightarrow 8,\langle 4\rangle \Rightarrow 6$ \\
$\{4,6\}$ & $1,2,9,10 \in \mathbb{E}^{3}$ & $\langle 1\rangle \Rightarrow 3,\langle 9\rangle \Rightarrow 7,\langle 10\rangle \Rightarrow 8,\langle 7\rangle \Rightarrow 5$ \\
$\{5,6\}$ & $1,2,9,10 \in \mathbb{E}^{3}$ & $\langle 1\rangle \Rightarrow 3,\langle 2\rangle \Rightarrow 4,\langle 9\rangle \Rightarrow 7,\langle 10\rangle \Rightarrow 8$ \\
\hline
\end{tabular}

We need to show that every tensegrity with a non-zero stress on every edge and with an underlying graph from Fig. 11 is in $\mathbb{E}^{3}$. For each graph, we follow a proof similar to the proof of Lemma 6, so we use the notation introduced following the lemma. The four vertices assumed to be in $\mathbb{E}^{3}$ are circled in Fig. 11. The proof is given in Table 1.

Thus, every stressed graph spans at most three dimensions.

Note that in each of the above cases, we did not use the fact that the strut had a nonzero stress. In fact, the two vertices incident to the strut have degree 4 , and our method of proving that a stressed graph spans three dimensions uses only degree 3 vertices. This will be helpful for dealing with the case where the strut and exactly one other edge have a zero stress.

Case 3: All edges have a non-zero stress except for two. If the two edges are incident to the same vertex not incident to the strut, then a degree 1 vertex is created. If neither edge is incident to vertex 3 or vertex 8 , then the resulting graph has six or fewer vertices. Since every vertex has either degree 3 or 4 , the resulting graph is either not complete or has fewer than six vertices. By Lemma 5 , the tensegrity is in $\mathbb{E}^{3}$. Also, if one of the two edges with a zero stress is the strut then the stressed graph spans three dimensions by the remarks at the end of Case 2. Thus, we are left with the cases where one of the removed edges is incident to either vertex 3 or 8 but is not the edge $\{3,8\}$. By symmetry, we can assume one of the removed edges is incident to vertex 3 . Tables $2-4$ deal with the possibilities where two edges are removed, where one of the edges is $\{1,3\},\{3,4\}$, or

Table 2. Two edges removed, including edge $\{1,3\}$.

\begin{tabular}{lccc}
\hline Edges removed & Vertices Forced Collinear & Number of remaining vertices & Figure \\
\hline$\{1,3\}$ and $\{2,4\}$ & $1,2,4,9,10$ & 5 & \\
$\{1,3\}$ and $\{2,10\}$ & $1,2,10$ & 7 & $\{1,3\}$ and $\{3,4\}$ \\
$\{1,3\}$ and $\{3,4\}$ & $1,3,7$ & 7 & $\{1,3\}$ and $\{3,4\}$ \\
$\{1,3\}$ and $\{3,5\}$ & $1,3,5$ & 7 & $\{1,3\}$ and $\{3,4\}$ \\
$\{1,3\}$ and $\{4,6\}$ & $1,4,6$ & 7 & $\{1,3\}$ and $\{3,4\}$ \\
$\{1,3\}$ and $\{5,6\}$ & $1,5,6$ & 7 & $\{1,3\}$ and $\{3,4\}$ \\
$\{1,3\}$ and $\{5,7\}$ & $1,5,7$ & 7 & $\{1,3\}$ and $\{5,7\}$ \\
$\{1,3\}$ and $\{7,8\}$ & 1,7 & 8 & \\
$\{1,3\}$ and $\{7,9\}$ & $1,7,9,2,10$ & 5 & \\
$\{1,3\}$ and $\{8,10\}$ & $1,8,10,2$ & 6 & \\
\hline
\end{tabular}


Table 3. Two edges removed, including edge $\{3,4\}$.

\begin{tabular}{lccc}
\hline Edges removed & Vertices Forced Collinear & Number of remaining vertices & Figure \\
\hline$\{3,4\}$ and $\{1,2\}$ & $1,2,4$ & 7 & $\{1,3\}$ and $\{2,10\}$ \\
$\{3,4\}$ and $\{1,9\}$ & $1,4,9$ & 7 & $\{1,3\}$ and $\{2,10\}$ \\
$\{3,4\}$ and $\{2,10\}$ & $4,2,10$ & 7 & $\{1,3\}$ and $\{2,10\}$ \\
$\{3,4\}$ and $\{3,5\}$ & $3,4,5$ & 7 & $\{1,3\}$ and $\{2,10\}$ \\
$\{3,4\}$ and $\{5,6\}$ & $4,5,6$ & 7 & $\{3,4\}$ and $\{5,6\}$ \\
$\{3,4\}$ and $\{5,7\}$ & $4,5,7$ & 7 & $\{3,4\}$ and $\{5,6\}$ \\
$\{3,4\}$ and $\{6,8\}$ & 4,6 & 8 & $\{3,4\}$ and $\{6,8\}$ \\
$\{3,4\}$ and $\{7,8\}$ & 4,7 & 8 & $\{3,4\}$ and $\{6,8\}$ \\
$\{3,4\}$ and $\{7,9\}$ & $4,7,9$ & 7 & $\{1,3\}$ and $\{3,4\}$ \\
$\{3,4\}$ and $\{8,10\}$ & 4,10 & 8 & $\{1,3\}$ and $\{7,8\}$ \\
$\{3,4\}$ and $\{9,10\}$ & $4,9,10$ & 7 & $\{1,3\}$ and $\{2,10\}$ \\
\hline
\end{tabular}

$\{3,5\}$. Note that if the resulting stressed graph has five vertices, then it has dimension at most three. Also, if the graph has six vertices, then by Lemma 5 it has dimension at most three (since each vertex has degree at most 4 , the stressed graph cannot be complete). Thus, if the number of remaining vertices is six or fewer, the graph is not included.

Figure 12 shows all the resulting graphs up to isomorphism (where the strut is not considered a special edge for the purposes of the isomorphism). Tables $2-4$ go through all of the cases, and for each case, reference the resulting graph in Fig. 12.

We need to show that all of the stressed graphs span a maximum of three dimensions. We use a proof similar to Lemma 6 . The vertices assumed to be in $\mathbb{E}^{3}$ are circled in the figures. The proof is given in Table 5 .

Case 4: There is exactly one vertex with zero stresses on all incident edges. By the symmetry of Fig. 5, we need only consider the cases where the vertex with zero stress is $1,2,3,4$, or 5 . Since there is only one vertex with all incident edges having a stress of zero and since that vertex has degree 3 in $C_{5} \times C_{2}$, if the stressed tensegrity is in $\mathbb{E}^{3}$, then the remaining vertex can be folded into $\mathbb{E}^{3}$. Thus, our goal is to show that the resulting stressed graphs must span at most three dimensions.

Table 4. Two edges removed, including edge $\{3,5\}$.

\begin{tabular}{lccc}
\hline Edges removed & Vertices Forced Collinear & Number of remaining vertices & Figure \\
\hline$\{3,5\}$ and $\{1,2\}$ & $1,2,5$ & 7 & $\{1,3\}$ and $\{2,10\}$ \\
$\{3,5\}$ and $\{1,9\}$ & $1,5,9$ & 7 & $\{1,3\}$ and $\{3,4\}$ \\
$\{3,5\}$ and $\{2,4\}$ & $2,4,5$ & 7 & $\{1,3\}$ and $\{3,4\}$ \\
$\{3,5\}$ and $\{2,10\}$ & $2,5,10$ & 7 & $\{1,3\}$ and $\{3,4\}$ \\
$\{3,5\}$ and $\{4,6\}$ & $4,5,6,7$ & 6 & \\
$\{3,5\}$ and $\{6,8\}$ & 5,6 & 8 & $\{3,4\}$ and $\{6,8\}$ \\
$\{3,5\}$ and $\{7,8\}$ & 5,7 & 8 & $\{3,4\}$ and $\{6,8\}$ \\
$\{3,5\}$ and $\{7,9\}$ & $5,7,9,6$ & 6 & \\
$\{3,5\}$ and $\{8,10\}$ & 5,10 & 8 & $\{3,5\}$ and $\{8,10\}$ \\
$\{3,5\}$ and $\{9,10\}$ & $5,9,10$ & 7 & $\{1,3\}$ and $\{2,10\}$ \\
\hline
\end{tabular}




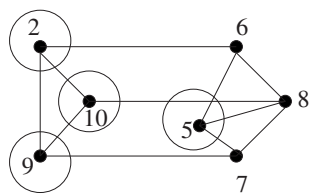

$\{1,3\}$ and $\{3,4\}$

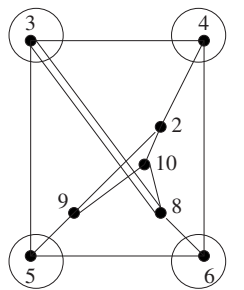

$\{1,3\}$ and $\{7,8\}$

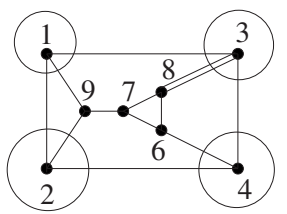

$\{3,5\}$ and $\{8,10\}$

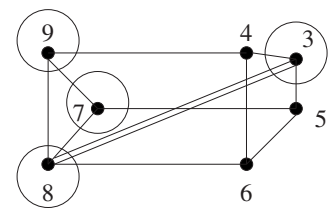

$\{1,3\}$ and $\{2,10\}$

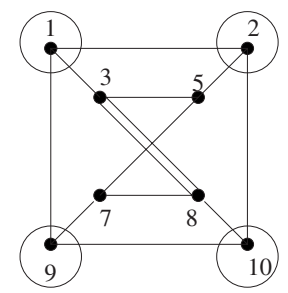

$\{3,4\}$ and $\{6,8\}$

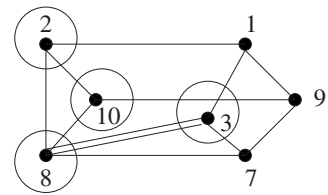

$\{3,4\}$ and $\{5,6\}$

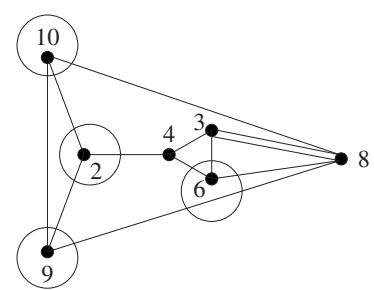

$\{1,3\}$ and $\{5,7\}$

Fig. 12. These graphs result from removing two edges. Each graph is isomorphic to several graphs obtained by removing two edges. The two edges given are one possibility, Tables $2-4$ give the other possibilities.

We start by examining the cases where the only edges with zero stress are the ones incident to the vertex in question. The other cases (where there are other edges with zero stress, but not additional vertices with all incident edges having a zero stress) will follow easily.

If the graph with the vertex removed has only six vertices, then by Lemma 5 the stressed graph can span at most $\mathbb{E}^{3}$. Thus, we do not need to draw the graphs for cases where the resulting graph has six or fewer vertices.

Table 5. Each of the stressed graphs for removing two edges (see Fig. 12) spans at most three dimensions.

\begin{tabular}{lll}
\hline$\{1,3\}$ and $\{3,4\}$ & $2,5,9,10 \in \mathbb{E}^{3}$ & $\langle 2\rangle \Rightarrow 6,\langle 10\rangle \Rightarrow 8,\langle 9\rangle \Rightarrow 7$ \\
$\{1,3\}$ and $\{2,10\}$ & $3,7,8,9 \in \mathbb{E}^{3}$ & $\langle 7\rangle \Rightarrow 5,\langle 9\rangle \Rightarrow 4,\langle 4\rangle \Rightarrow 6$ \\
$\{1,3\}$ and $\{7,8\}$ & $3,4,5,6 \in \mathbb{E}^{3}$ & $\langle 3\rangle \Rightarrow 8,\langle 4\rangle \Rightarrow 2,\langle 5\rangle \Rightarrow 9,\langle 9\rangle \Rightarrow 10$ \\
$\{3,4\}$ and $\{5,6\}$ & $2,3,8,10 \in \mathbb{E}^{3}$ & $\langle 2\rangle \Rightarrow 1,\langle 10\rangle \Rightarrow 9,\langle 3\rangle \Rightarrow 7$ \\
$\{3,4\}$ and $\{6,8\}$ & $1,2,9,10 \in \mathbb{E}^{3}$ & $\langle 1\rangle \Rightarrow 3,\langle 2\rangle \Rightarrow 5,\langle 9\rangle \Rightarrow 7,\langle 10\rangle \Rightarrow 8$ \\
$\{3,5\}$ and $\{8,10\}$ & $1,2,3,4 \in \mathbb{E}^{3}$ & $\langle 1\rangle \Rightarrow 9,\langle 3\rangle \Rightarrow 8,\langle 4\rangle \Rightarrow 6,\langle 6\rangle \Rightarrow 7$ \\
$\{1,3\}$ and $\{5,7\}$ & $2,6,9,10 \in \mathbb{E}^{3}$ & $\langle 2\rangle \Rightarrow 4,\langle 9\rangle \Rightarrow 8,\langle 6\rangle \Rightarrow 3$
\end{tabular}


Table 6. The possibilities for removing one vertex.

\begin{tabular}{cccc}
\hline Edges removed & Vertices Forced Collinear & Number of remaining vertices & Figure \\
\hline 1 & 2,9 & 7 & 13 \\
2 & $1,4,10$ & 6 & \\
3 & $1,4,5$ & 6 & \\
4 & 2,6 & 7 & 13 \\
5 & 6,7 & 7 & 13 \\
\hline
\end{tabular}

Table 6 provides the relevant information: the removed vertex, the vertices forced collinear (these are the vertices adjacent to the removed vertex), and the number of vertices in the resulting stressed graph (ignoring the vertices collinear with their neighbors). The resulting graphs (with more than six vertices) are shown in Fig. 13.

Now, we need that every tensegrity with a graph from Fig. 13 and with a non-zero stress spans $\mathbb{E}^{3}$. Since there is only one vertex with all incident edges having a stress of zero and since that vertex has degree 3 in $C_{5} \times C_{2}$, if the stressed tensegrity is in $\mathbb{E}^{3}$, then the remaining vertex can be folded into $\mathbb{E}^{3}$.

All three graphs from Fig. 13 have the same underlying graph as a graph from Case 1 (see Fig. 12). The graph for removing vertex 1 is the same as the graph obtained by removing edges $\{1,3\}$ and $\{2,10\}$; the graph for removing vertex 4 is the same as the graph obtained by removing edges $\{1,3\}$ and $\{3,4\}$; and the graph for removing vertex 5 is the same as the graph obtained by removing edges $\{3,4\}$ and $\{5,7\}$. Thus, as proved earlier, all three span at most $\mathbb{E}^{3}$ if there is a stress that is non-zero on every edge.

Finally, we need to deal with the case where an additional edge has a zero stress. If any of the graphs in Fig. 13 have a stress of zero on one of the edges, then an incident vertex becomes collinear with its neighbors, which allows us to ignore that vertex. Thus, if one of the edges has a stress of zero, the stressed tensegrity has at most six vertices and is in $\mathbb{E}^{3}$ by Lemma 5 .

Case 5: There are exactly two vertices with zero stress on all incident edges. We consider all possible pairs of vertices, using the symmetry of the graph to reduce the number of cases slightly. With two vertices removed, we would like the resulting graph to span at most two dimensions. Then one of the removed vertices forms three dimensions and the second can be folded into three dimensions. Note that if the resulting graph has five or fewer vertices, then by Lemma 5 it spans at most two dimensions (the graph will not be complete since only two vertices in $C_{5} \times C_{2}$ plus a strut have degree 4 ). We will show

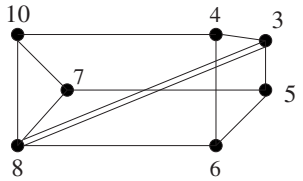

vertex 1

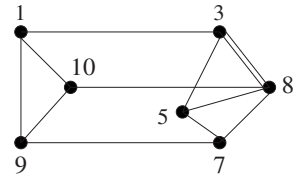

vertex 4

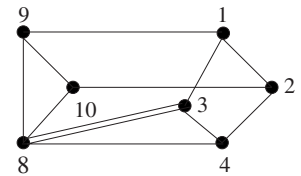

vertex 5

Fig. 13. Graphs resulting from removing one vertex-see Table 6 . 

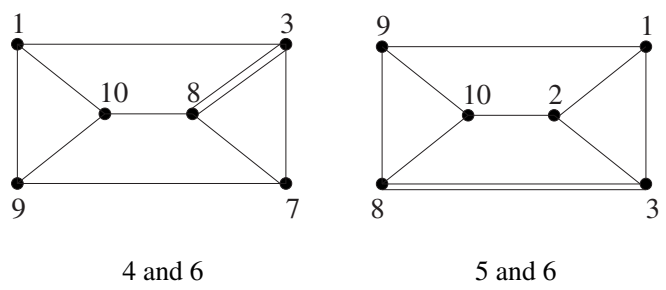

Fig. 14. Graphs for removing two vertices.

that up to isomorphism the only resulting graphs with more than five vertices are the two graphs in Fig. 14.

Let the two removed vertices be $a$ and $b$. Note that if $a$ or $b$ is adjacent to a vertex other than 3 or 8 (the two vertices incident to the strut), then that vertex must be collinear with its neighbors. Also, if $a$ and $b$ are both adjacent to the same vertex other than 3 or 8 , then that vertex must also have zero stress on all adjacent vertices (see Case 6). If $a$ and $b$ are both adjacent to either 3 or 8 then that vertex must be collinear with its neighbors.

We will show that unless $a$ and $b$ are adjacent with both $a$ and $b$ adjacent to either 3 or 8 , the resulting stressed graph has at most five vertices.

First, suppose that $a$ and $b$ are not adjacent. Then each is adjacent to three vertices (or four vertices if $a$ or $b$ is 3 or 8). We assume that each is adjacent to three vertices, since more adjacent vertices will create even more vertices forced collinear. There could be six distinct vertices among the neighbors with 3 or 8 allowed as a neighbor (creating at least four vertices forced collinear), or there could be five distinct vertices among the neighbors with either 3 and 8 duplicated (again creating at least four vertices forced collinear-this time one of the vertices forced collinear is 3 or 8 ), or there could be four distinct vertices with both 3 or 8 duplicated (again creating at least four vertices forced collinear). Thus, there are at least four vertices forced collinear. With four vertices forced collinear and $a$ and $b$ removed, the resulting stressed graph has at most four vertices and spans at most two dimensions. Thus, $a$ and $b$ can fold into three dimensions.

Next, suppose that $a$ and $b$ are adjacent with $a$ not adjacent to 3 or 8 (and $b$ possibly adjacent to 3 or 8 ). Note that $b$ cannot be adjacent to both 3 and 8 , since there are no triangles in $C_{5} \times C_{2}$ plus a strut. Since $a$ and $b$ are adjacent, they cannot be adjacent to the same vertex as that would form a triangle. Vertex $a$ must be adjacent to two vertices that are forced collinear (since it is not adjacent to 3 or 8). Vertex $b$ must be adjacent to at least one vertex that is forced collinear. With three vertices forced collinear and $a$ and $b$ removed, the resulting stressed graph has at most five vertices and spans at most two dimensions. Thus, $a$ and $b$ can fold into three dimensions.

Thus, we must have $a$ and $b$ adjacent with both $a$ and $b$ adjacent to either 3 or 8 . The only possibilities, then, for the two removed vertices are 4 and 6,5 and 6 , and 5 and 7 . From the second drawing in Fig. 5 we can see that removing 4 and 6 is symmetric to removing 5 and 7 . Thus, we only need to deal with removing 4 and 6 and removing 5 and 6. The two resulting graphs are in Fig. 14.

Now, we need to show that the resulting stressed tensegrities in Fig. 14 are in $\mathbb{E}^{2}$. Then the tensegrity plus one of the vertices with no adjacent non-zero stress is in $\mathbb{E}^{3}$, and the final vertex with no adjacent non-zero stress can be folded into $\mathbb{E}^{3}$. 
If any edge of the above graphs has a zero stress, then one of the incident vertices is collinear with its neighbors. In that case we effectively have a graph with one fewer vertex. All such graphs have at most five vertices, and by Lemma 5 are in $\mathbb{E}^{2}$.

We just need to show that the two resulting graphs in Fig. 14 are all in $\mathbb{E}^{2}$, assuming non-zero stress on all edges. The graphs for these two tensegrities are all the same: the only difference is the location of the strut. We will show that the graph with vertices 4 and 6 removed is in $\mathbb{E}^{2}$. The same argument applies to the other tensegrity by a renumbering of vertices.

We use the notation introduced after Lemma 6; this time we are showing that the graph is in $E^{2}$. We assume that vertices 1,2 , and 10 are in $E^{2}$. Then the remaining vertices can by shown to be in $E^{2}:\langle 1\rangle \Rightarrow 3,\langle 9\rangle \Rightarrow 7,\langle 10\rangle \Rightarrow 8$. Thus, the tensegrity is in $E^{2}$.

Case 6: There are exactly three vertices with zero stress on all incident edges. Let $a, b$, and $c$ denote the three vertices with zero stress on all incident edges. Since there are no triangles in the graph of $C_{5} \times C_{2}$ plus an edge, at least two of $a, b$, and $c$ are non-adjacent. Thus, we can assume that $b$ and $c$ are non-adjacent. We will show that the resulting graphs for removing these three vertices have four or fewer vertices, and therefore lie in at most two dimensions. Including vertex $a$ makes at most three dimensions. Vertices $b$ and $c$ can then be folded into dimension three, because both vertices are only adjacent to vertices in $\mathbb{E}^{3}$ (since they are not adjacent to each other).

We just need to show that graphs resulting from the removal of three vertices have at most four vertices. Vertex $a$ is adjacent to at least one vertex other than $b$ and $c$. Vertices $b$ and $c$ are both adjacent to at least two vertices other than $a$. Thus, there are five vertices that are adjacent to $a, b$, and $c$ (some vertices may be double counted). If a vertex of degree 3 is adjacent to $a, b$, or $c$, then it is forced collinear with its other neighbors. If a vertex of degree 4 (vertex 3 or 8 ) is adjacent to two of $a, b$, and $c$, then it is forced collinear with its other neighbors. We need to determine how many of these five vertices are forced collinear.

If a vertex other than vertex 3 or 8 is adjacent to $a, b$, or $c$, then it is collinear with its neighbors and, thus, not in the resulting graph. If vertex 3 or 8 is adjacent to two of $a$, $b$, and $c$, then it becomes collinear with its neighbors and not in the resulting graph. If a vertex other than 3 or 8 is adjacent to two of $a, b$, and $c$, then it has zero stress on all incident edges (see Case 5). If vertex 3 or 8 is adjacent to all three of $a, b$, and $c$, then it has zero stress on all incident edges (see Case 5). We had five vertices adjacent to $a$, $b$, and $c$. Vertices 3 and 8 are allowed to be in this list of five once and remain in the resulting graph. Thus, removing $a, b$, and $c$ forces at least three $(5-2)$ other vertices to become collinear with neighbors. This leaves at most four vertices in the resulting graph.

Case 7: There are exactly four vertices with zero stress on all incident edges. By an argument similar to Case 6, the resulting stressed graph (after dealing with vertices of degree 2) has at most four vertices. The case where the stressed graph has four vertices is precisely when vertices $1,2,9$, and 10 are the ones removed. This case was dealt with in Lemma 9. In all other cases, the resulting graph contains a collinear cycle (see Case 1).

Case 8: Additional cases. We need to show that we have considered all relevant cases. We could remove more edges without removing a vertex (similar to Cases 2 and 3). 
However, if three or more edges have zero stress, the resulting graph either has at least one vertex with zero stress on all adjacent vertices (see Cases 4-7) or has six or fewer vertices (in $\mathbb{E}^{3}$ by Lemma 5).

We could remove more vertices as in Cases 4-7. However, if a graph has any more vertices removed, then the resulting graph has a cycle which is forced collinear by the stress (Case 1).

Thus, we have considered all cases, so $C_{5} \times C_{2}$ is 3-realizable.

\section{Acknowledgements}

I thank Robert Connelly for many helpful comments and suggestions. In particular, he suggested Theorem 3 and Lemma 1. Additionally, I thank the two anonymous referees for their many helpful suggestions.

\section{References}

[BC1] Belk, Maria; Connelly, Robert, Realizability of graphs, Discrete Comput. Geom., this issue, pp. 125137.

[BC2] Bezdek, Károly; Connelly, Robert, Two-distance preserving function from Euclidean space. Discrete geometry and rigidity (Budapest, 1999). Period. Math Hungar. 39(1-3), (1999), 185-200, MR1783825.

[BPR] Basu, Saugata; Pollack, Richard; Roy, Marie-Françoise, Algorithms in Real Algebraic Geometry, Algorithms and Computation in Mathematics, 10, Springer-Verlag, Berlin, 2003, MR1998147.

[C] Connelly, Robert, Rigidity and energy, Invent. Math. 66(1) (1982), 11-33, MR0652643.

[CW] Connelly, Robert; Whiteley, Walter, Second-order rigidity and prestress stability for tensegrity frameworks, SIAM J. Discrete Math. 9(3) (1996), 453-491, MR1402190.

[D] Diestel, Reinhard, Graph Theory, second edition, Graduate Texts in Mathematics, 173, Springer-Verlag, New York, 2000, MR1743598.

[M] Maple 8 for Linux, Maplesoft Inc., http://www.maplesoft.com.

[R] Rudin, Walter, Principles of Mathematical Analysis, third edition, International Series in Pure and Applied Mathematics, McGraw Hill, New York, 1976, MR0385023.

[RW] Roth, Ben; Whiteley, Walter, Tensegrity frameworks, Trans. Amer. Math. Soc. 265(2) (1981), 419-446, MR0610958.

Received October 11, 2004, and in revised form June 4, 2006. Online publication February 9, 2007. 